\title{
Does the Unexpected Presence of Non-organ-confined Disease at Final Pathology Undermine Cancer Control in Patients with Clinical T1N0M0 Renal Cell Carcinoma Who Underwent Partial Nephrectomy?
}

\author{
Umberto Capitanio $^{a, b, *}$, Grant D. Stewart ${ }^{c, \dagger}$, Tobias Klatte $^{d}$, Bulent Akdogan $^{e}$, Marco Roscigno $^{f}$, \\ Martin Marszalek ${ }^{g}$, Paolo Dell'Oglio ${ }^{a, b}$, Emanuele Zaffuto $^{a, b}$, Oscar Rodriguez Faba $^{h}$, \\ Maciej Salagierski ${ }^{i}$, James Lingard $^{c}$, Marco Carini $^{j}$, Idir Ouzaid $^{k}$, Maria Carmen Mir ${ }^{l}$, \\ Francesco Montorsi $^{a, b}$, Luigi Filippo Da Pozzo ${ }^{f}$, Christian Stief ${ }^{m}$, Andrea Minervini ${ }^{j}$, \\ Sabine D. Brookman-May ${ }^{m, n}$ \\ ${ }^{a}$ Unit of Urology, University Vita-Salute, San Raffaele Scientific Institute, Milan, Italy; ${ }^{\mathrm{b}}$ Division of Experimental Oncology, Urological Research Institute, \\ IRCCS San Raffaele Scientific Institute, Milan, Italy; ${ }^{\circ}$ Edinburgh Urological Cancer Group, University of Edinburgh, Western General Hospital, Edinburgh, UK; \\ ${ }^{\mathrm{d}}$ Medical University of Vienna, Vienna, Austria; ${ }^{\mathrm{e}}$ Hacettepe University, Ankara, Turkey; ${ }^{\mathrm{f}}$ Papa Giovanni XXIII Hospital, Bergamo, Italy; ${ }^{\mathrm{g}}$ Donauspital, Vienna, \\ Austria; ${ }^{\mathrm{h}}$ Fundacio Puigvert, Barcelona, Spain; ${ }^{\mathrm{i}}$ Kent \& Canterbury Hospital, Canterbury, UK; ${ }^{\mathrm{j}}$ Azienda Ospedaliero Universitaria Careggi, Florence, Italy; \\ ${ }^{\mathrm{k}}$ Bichat University Hospital, Paris, France; ${ }^{1}$ Hospital del Mar-Parc de Salut Mar-IMIM, Barcelona, Spain; ${ }^{\mathrm{m}}$ Department of Urology, Ludwig-Maximilians \\ University Munich, Campus Grosshadern, Munich, Germany; ${ }^{\mathrm{n}}$ Janssen Pharma Research and Development, Los Angeles, CA, USA
}

Article info

Associate Editor: James Catto

Keywords:

Renal cancer

Kidney cancer

Partial nephrectomy

Radical nephrectomy

Cancer control

Upstaging

\begin{abstract}
Background: A non-negligible proportion of individuals diagnosed with cT1 renal cell carcinoma (RCC) are upstaged to pT3a at final pathology. Few data on oncological outcomes for these patients are available to determine whether partial nephrectomy (PN) might jeopardise cancer control.

Objective: To assess, within an international multi-institutional collaboration, whether PN might undermine cancer control relative to radical nephrectomy $(\mathrm{RN})$ in RCC patients with unexpected pT3a disease.

Design, setting, and participants: International multi-institutional collaboration including patients with cT1abNOM0-pT3a RCC. Intervention: $\mathrm{PN}$ or $\mathrm{RN}$.

Outcome measurements and statistical analysis: We used Kaplan-Meier analyses, before and after propensity-score matching, to evaluate differences in metastatic progression (MP) and cancer-specific mortality (CSM) rates during follow-up. Univariable and multivariable Cox regression analyses were used to assess predictors of MP and CSM.

Results and limitations: Overall, 309 patients with cT1abNOM0 RCC (cT1aNOM0, $n=107$, 34.6\%; cT1bN0M0, $n=202,65.4 \%$ ) had pT3a disease according to final pathology. Patients were treated with either PN $(n=71,23 \%)$ or RN $(n=238,77 \%)$. MP at 1,2 , and $5 \mathrm{yr}$ was detected in $9.1 \%, 13.3 \%$, and $24.1 \%$ of patients, respectively. CSM was $3.5 \%$, $10.7 \%$, and $18.4 \%$ at 1,2 , and $5 \mathrm{yr}$, respectively. After matching, no difference in terms of

$1{ }^{\dagger}$ Current affiliation: Academic Urology Group, University of Cambridge, Addenbrooke's Hospital, Cambridge, UK.

* Corresponding author. Unit of Urology, IRCCS San Raffaele Scientific Institute, Via Olgettina 60 20132 Milan, Italy. Tel.: +3902 26437286; Fax: +39 0226437298.

E-mail address: umbertocapitanio@gmail.com (U. Capitanio).
\end{abstract}

http://dx.doi.org/10.1016/j.euf.2017.02.020

2405-4569/@ 2017 European Association of Urology. Published by Elsevier B.V. All rights reserved. 
MP or CSM was observed between the PN and RN cohorts (both $p>0.3$ ). On multivariable analysis, type of surgery (PN vs RN) was not an independent predictor of either MP $(p=0.3)$ or CSM $(p=0.4)$. Limitations include the retrospective design.

Conclusions: In patients with unexpected pT3a RCC at final pathology, PN does not appear to jeopardise cancer control with regard to MP and CSM.

Patient summary: Cancer control is similar between patients treated with removal of the entire kidney and those with only partial removal, even if the final histology examination demonstrates a tumour that is unexpectedly not confined within the kidney.

(C) 2017 European Association of Urology. Published by Elsevier B.V. All rights reserved.

\section{Introduction}

The majority of patients currently diagnosed with renal cell carcinoma (RCC) harbour small, organ-confined tumours [1]. In this setting, international urological guidelines recommend different management options, ranging from surveillance to nephron-sparing surgery, such as partial nephrectomy (PN) [2-4]. For small renal masses, PN reduces the risk of cardiovascular morbidity [5] and is associated with better functional outcomes [6-10] compared to radical nephrectomy (RN) without compromising cancer control [11]. However, a non-negligible proportion of individuals treated with PN show invasion of perirenal sinus fat tissue or involvement of the inner renal blood vessels at final pathology and thus are upstaged to stage pT3a [1214]. The clinical impact of such upstaging is debated. In addition, only a few small studies have formally evaluated the impact of surgical approach (PN vs RN) on oncological outcomes among patients with cT1 disease who experienced upstaging to pT3a, and these comprise extremely limited patient cohorts [12-14].

To bridge this gap, we used the largest multi-institutional cohort of patients with clinical T1abNOM0 RCC treated with PN or RN for whom final pathology revealed unexpected pT3a disease to investigate the hypothesis that $\mathrm{PN}$ does not compromise cancer control relative to $\mathrm{RN}$ in this subset of patients.

\section{Patients and methods}

\subsection{Study population}

For our retrospective analysis we used a multi-institutional database of surgically treated RCC patients from ten European tertiary care centres. Specifically, the study cohort consisted of individuals diagnosed with RCC and treated with either RN or PN between 1988 and 2015. All individuals were preoperatively staged as CT1NOMO and were staged as PT3a at final pathology. Individuals with bilateral kidney cancer or with monolateral multifocal tumours were not considered.

\subsection{Clinical and pathological evaluation}

TNM stages were assigned according to the 2009 American Joint Committee on Cancer/Union Internationale Contre le Cancer classification [2]. Cases before the introduction of the most recent classification scheme were reclassified. Clinical tumour size was based on preoperative imaging and defined as the greatest tumour diameter in centimetres. Stage cT1 was defined in accordance with the 2009 TNM classification for RCC: tumour of $\leq 7 \mathrm{~cm}$ in the greatest dimension, limited to the kidney, and without radiographic signs of extension to the perinephric tissue or the major veins [2]. Surgery was performed within 3 mo from clinical staging. All specimens were evaluated by an experienced uropathologist at each treating institution. Patients were evaluated at 3 mo after surgery and subsequently according to individualised follow-up schedules comprising at least two annual visits.

\subsection{Outcomes}

The joint primary outcomes were metastatic progression (MP) and cancer-specific mortality (CSM). MP was defined as retroperitoneal nodal recurrence or systemic recurrence (skeletal and/or visceral relapse) at imaging during follow-up. Patients who died from RCC were defined as having CSM. CSM was defined by the attending urologist or oncologist who followed the patient and/or from information on the death certificate.

\subsection{Statistical analyses}

Descriptive statistics were used to categorise the baseline characteristics among patients treated with either PN or RN. Frequencies and proportions were reported for categorical variables. Mean, medians, and interquartile ranges were reported for continuous variables. Mann-Whitney $U$ tests and $\chi^{2}$ tests were applied to assess the statistical significance of differences in medians and proportions, respectively.

The effect of treatment type (PN vs RN) on MP and CSM was assessed in the overall cohort. Actuarial survival rates at various time points after PN and RN were calculated.

To account for possible differences between the two groups that might be related to differences in the surgical approach, propensityscore matching was performed. Specifically, propensity scores were computed using a logistic regression model that evaluated the odds of receiving PN versus RN according to clinical tumour size, age, and year of surgery. The nearest-neighbour method, with a calliper width of 0.2 times the standard deviation of the logit and a 1-to-1 matching ratio, was used. After matching, Kaplan-Meier plots were used to graphically depict the survival rates observed for the matched cohorts. Differences in the rates of MP and CSM were tested using the log-rank test.

To test the hypothesis that treatment type (PN vs RN) may affect the risk of MP and CSM after surgery, multivariate Cox regression analyses were performed in the unmatched cohort after adjusting for all clinical and pathological covariates.

All statistical tests were two-sided with a level of significance set at $p<0.05$. Analyses were performed using SPSS version 20 (IBM Corp., Somers, NY, USA) for statistical computing and the R software environment for graphics (version 3.3.0; www.r-project.org).

\section{Results}

Overall, among 3863 patients with a clinically defined diagnosis of T1abN0M0 RCC, 309 (8\%) harboured pT3a 
disease at final pathology (cT1aN0M0, $n=107,34.6 \%$; cT1bN0M0, $n=202,65.4 \%)$. Patients were treated with either PN ( $n=71,23 \%)$ or $\mathrm{RN}(n=238,77 \%)$. Relative to $\mathrm{RN}, \mathrm{PN}$ cases were treated more recently $(p<0.001$, Table 1$)$ and had smaller masses (median tumour size 3.0 vs $5.5 \mathrm{~cm}$, $p<0.001$ ) and a lower proportion of high Fuhrman grade (G3-4 29.6 vs 47.1\%). An open, laparoscopic, and robotic approach was used in $72.9 \%, 2.8 \%$, and $24.3 \%$ of PN cases, and $60.7 \%, 36.5 \%$, and $2.8 \%$ of RN cases, respectively. No significant differences were observed between the PN and RN groups with regard to patient age and the presence of necrosis or sarcomatoid features (Table 1). pT3a was defined according to the presence of perirenal fat invasion only in $82.1 \%$ of PN and $43.6 \%$ of RN patients ( $p<0.001$; Table 1$)$. Only six patients $(1.9 \%)$ had positive surgical margins at final pathology ( 0.8 vs $5.6 \%$ for RN vs PN; $p=0.01)$.

After mean follow-up of 52 mo ( 55 mo for RN vs 43 mo for PN), local recurrence was recorded in six RN cases (2.8\%) and two PN cases (2.9\%). MP at 1, 2, and 5 yr was observed in $9.1 \%, 13.3 \%$, and $24.1 \%$ of cases, respectively (Fig. 1 A). CSM was $3.5 \%, 10.7 \%$, and $18.4 \%$ at 1,2 , and $5 \mathrm{yr}$, respectively
(Fig. 2A). After matching, no differences in MP and CSM were observed between the PN and RN groups (both $p>0.3$; Figs. $1 \mathrm{~B}$ and $2 \mathrm{~B}$ ).

In multivariable analyses for MP prediction, clinical tumour size (hazard ratio [HR] 1.4, 95\% confidence interval [CI] 1.0-1.8; $p=0.02$ ) and sarcomatoid features (HR 4.3, 95\% CI $1.1-16.3 ; p=0.003)$ were independent predictors. In multivariable analyses for CSM prediction, age at surgery (HR 1.1, 95\% CI 1.0-1.1; $p=0.005$ ) and clinical tumour size (HR 1.4, 95\% CI 1.1-1.9; $p=0.03$ ) were independent predictors. Type of surgery (PN vs RN) was not an independent predictor of either MP ( $p=0.3)$ or CSM ( $p=0.4$; Table 2$)$.

\section{Discussion}

International guidelines recommend that RCC patients with organ-confined tumours should undergo PN whenever technically feasible, which allows adequate cancer control and the preservation of functional parenchyma [2-4,15]. The benefits of this approach in terms of the general health of patients during the follow-up period have been well

Table 1 - Clinical and pathological characteristics of 309 patients treated with partial nephrectomy (PN) or radical nephrectomy (RN) for clinical cT1abNOMO renal cell carcinoma for whom pathology revealed unexpected pT3a disease.

\begin{tabular}{|c|c|c|c|}
\hline Variable & $\mathrm{PN}(\mathrm{n}=71,23 \%)$ & $\mathrm{RN}(\mathrm{n}=238,77 \%)$ & $p$ value \\
\hline \multicolumn{4}{|l|}{ Clinical characteristics } \\
\hline Age (yr) & $67(60-76)$ & $66(58-74)$ & 0.6 \\
\hline Year of surgery & $2010(2006-2012)$ & 2006 (2001-2009) & $<0.001$ \\
\hline Tumor size $(\mathrm{cm})$ & $3.0(2.2-4.4)$ & $5.5(4.2-6.5)$ & $<0.001$ \\
\hline Clinical stage, $n(\%)$ & & & $<0.001$ \\
\hline cT1aNOMO & $53(74.6 \%)$ & $54(22.7 \%)$ & \\
\hline cT1bNOM0 & $18(25.4 \%)$ & $184(77.3 \%)$ & \\
\hline \multicolumn{4}{|l|}{ Pathological features } \\
\hline pT3a subclassification (\%) & & & $<0.001$ \\
\hline PFI only & 82.1 & 43.6 & \\
\hline SFI only & 14.9 & 24.1 & \\
\hline $\mathrm{PFI}+\mathrm{SFI}$ or $\mathrm{PFI} / \mathrm{SFI}+\mathrm{RVI}$ & 3.0 & 32.3 & \\
\hline Surgical margins (\%) & 5.6 & 0.8 & 0.01 \\
\hline Fuhrman grade 3-4 (\%) & 29.6 & 47.1 & 0.009 \\
\hline Sarcomatoid features (\%) & 1.5 & 1.8 & 0.8 \\
\hline Necrosis (\%) & 26.1 & 37.6 & 0.1 \\
\hline
\end{tabular}

Table 2 - Multivariable Cox regression analyses predicting metastatic progression and cancer-specific mortality.

\begin{tabular}{|c|c|c|c|c|}
\hline \multirow[t]{2}{*}{ Variable } & \multicolumn{2}{|c|}{ Metastatic progression } & \multicolumn{2}{|c|}{ Cancer-specific mortality } \\
\hline & $\mathrm{HR}(95 \% \mathrm{CI})$ & $p$ value & $\mathrm{HR}(95 \% \mathrm{CI})$ & $p$ value \\
\hline Age & $1.0(1.0-1.1)$ & 0.07 & $1.1(1.0-1.1)$ & 0.005 \\
\hline Year of surgery & $1.0(0.9-1.0)$ & 0.6 & $1.0(0.9-1.1)$ & 0.5 \\
\hline Tumour size & $1.4(1.0-1.8)$ & 0.02 & $1.4(1.0-1.9)$ & 0.03 \\
\hline Fuhrman grade 3-4 & $1.9(0.9-3.8)$ & 0.08 & $1.8(0.8-4.2)$ & 0.2 \\
\hline Sarcomatoid features & $4.3(1.1-16.3)$ & 0.03 & $4.7(0.8-25.0)$ & 0.07 \\
\hline Necrosis & $1.1(0.6-2.3)$ & 0.7 & $1.2(0.5-2.7)$ & 0.7 \\
\hline pT3a subclassification & & 0.2 & & 0.7 \\
\hline SFI only vs PFI only & $0.9(0.3-2.3)$ & & $0.6(0.2-2.0)$ & \\
\hline $\mathrm{PFI}+\mathrm{SFI}$ or PFI/SFI + RVI vs PFI only & $1.7(0.8-3.6)$ & & $0.9(0.4-2.3)$ & \\
\hline Surgical margins & $2.3(0.3-18.4)$ & 0.9 & $2.7(0.4-15.1)$ & 0.3 \\
\hline \multicolumn{5}{|l|}{ Treatment type } \\
\hline PN vs RN & $0.5(0.1-1.8)$ & 0.3 & $0.6(0.1-2.3)$ & 0.4 \\
\hline
\end{tabular}



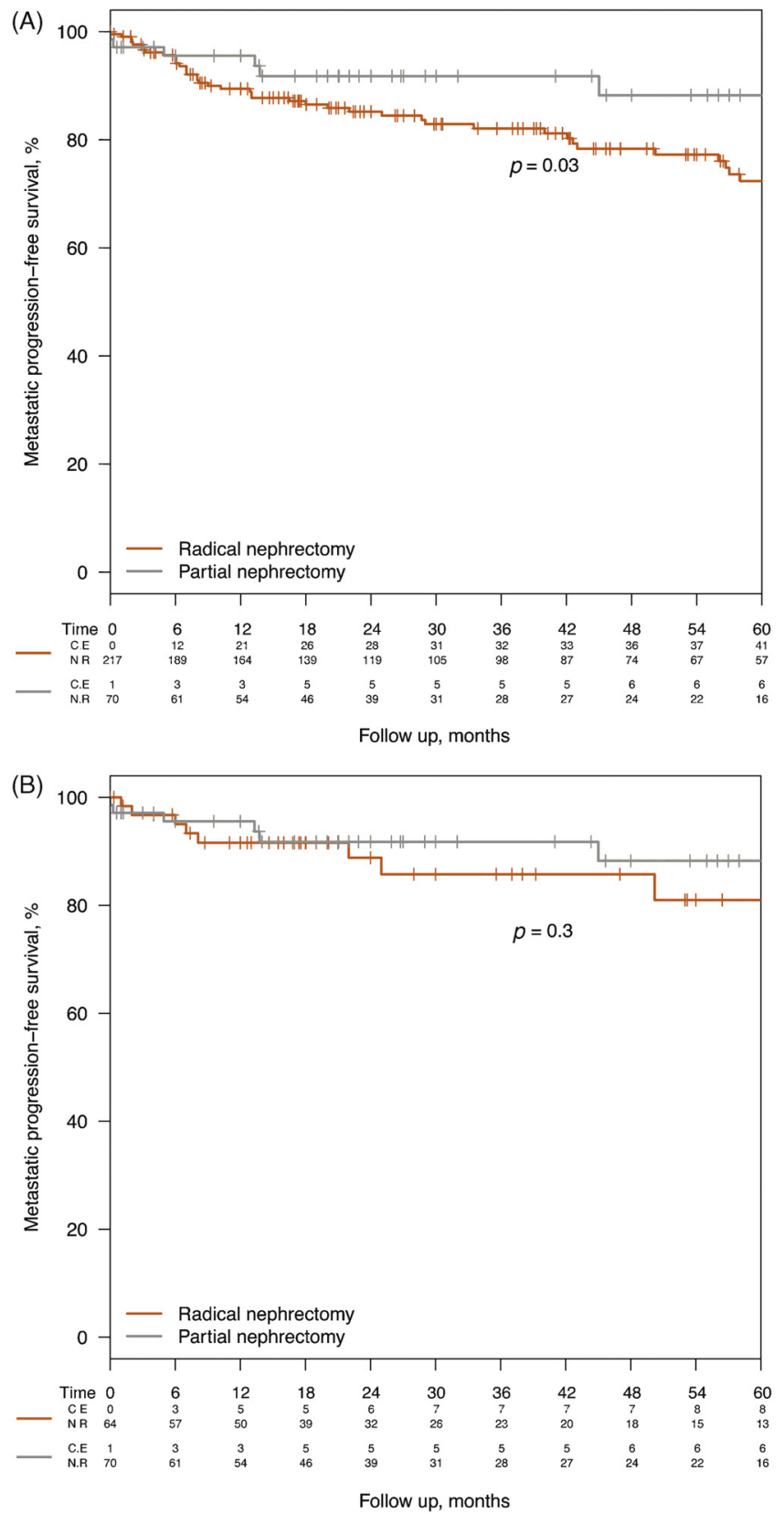

Fig. 1 - Kaplan-Meier analyses of metastatic progression-free survival according to surgical treatment (partial vs radical nephrectomy) (A) before and $(B)$ after propensity-score matching. $\mathrm{CE}=$ cumulative events; $\mathrm{NR}=$ number at risk.

established [5-11]. However, preoperative assessment of clinical stage may sometimes underestimate the actual tumour burden, leading to a pathological upstaging with potential detrimental effects on a patient's prognosis in relation to pathological tumour stage [16]. For approximately $10-20 \%$ of patients with disease staged as cT1NOM0, final pathology reveals unexpected non-organ-confined disease (pT3a) owing to the presence of microscopic invasion of the renal vein and/or of the perirenal/sinus fat $[14,17,18]$. Computed tomography imaging has sensitivity of $59-88 \%$ and specificity of $71-93 \%$ in predicting the presence of pT3 disease [19]. It has been suggested that cancer
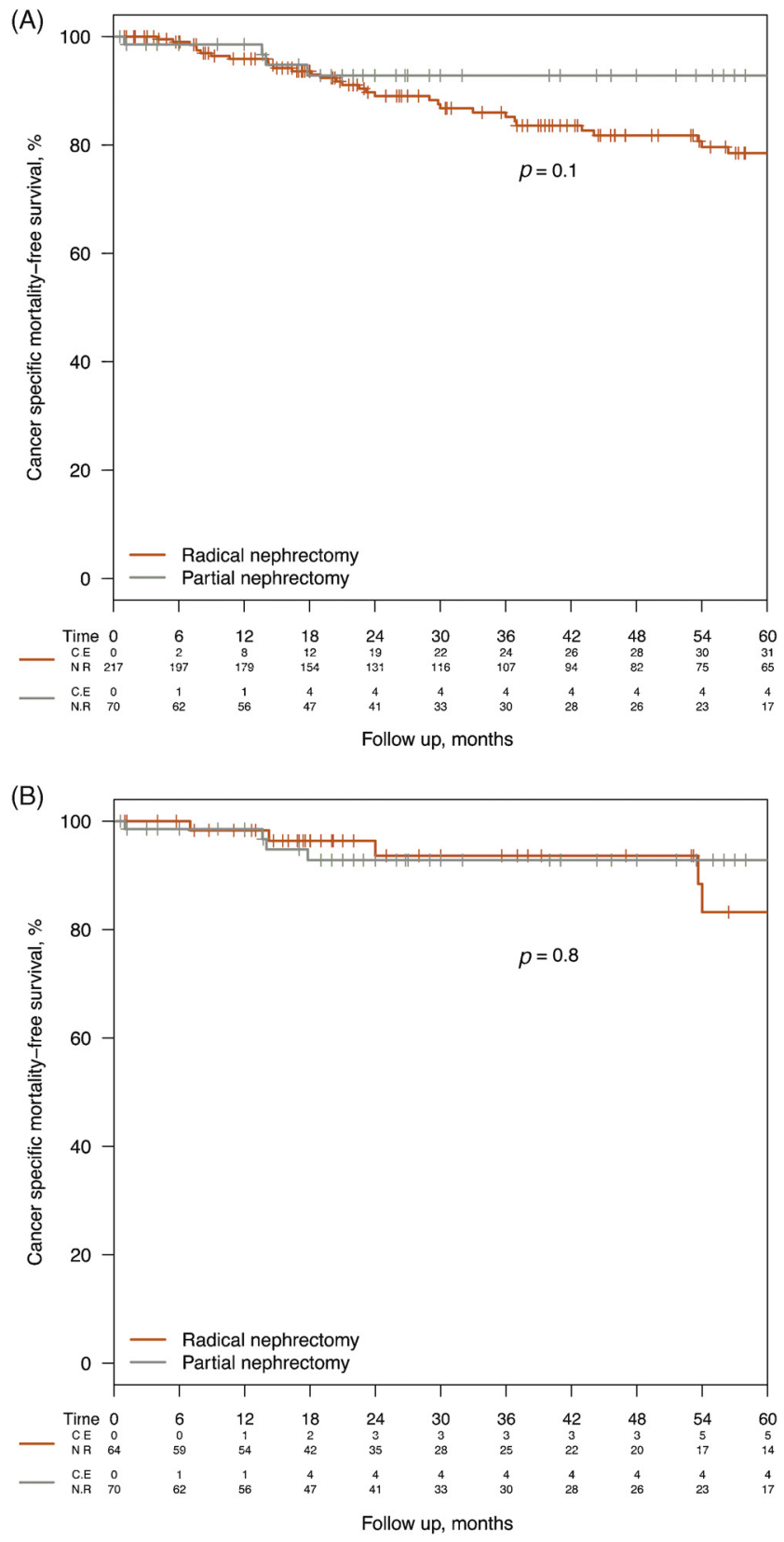

Fig. 2 - Kaplan-Meier analyses of cancer-specific mortality-free survival according to surgical treatment (partial vs radical nephrectomy) (A) before and $(B)$ after propensity-score matching. $C E$ = cumulative events; $\mathrm{NR}=$ number at risk.

control could be jeopardised relative to $\mathrm{RN}$ for this pT3 upstaging in patients who have undergone PN [12-14,20].

Many reports have already assessed potential predictors of unfavourable characteristics at final pathology in patients treated with PN [18,20,21]. For instance, Gorin and colleagues [13] evaluated the early oncological endpoint of recurrence-free survival (RFS) in patients with RCC upstaged from cT1 to pT3a, but only in the specific setting of robotic PN. Only 41 patients (4.8\%) were upstaged to pT3a. The 24-mo RFS estimates for cT1 and pT3a tumours were $99.2 \%$ and $91.8 \%$, respectively $(p=0.003)$. Factors associated with tumour upstaging included high tumour complexity 
(nephrometry score), increasing preoperative tumour diameter, and hilar location [13]. Ball et al. [18] investigated predictors associated with adverse pathology, defined as high pathological grade and/or pT3a disease, among $771 \mathrm{cT} 1$ RCC cases. Male gender, tumour size $>4 \mathrm{~cm}$, and high tumour complexity score were independent predictors of adverse pathology following PN. These studies and others focused on upstaging only; however, very few data are available for comparison of cancer control between PN and RN in upstaged patients. Nayak et al. [14] assessed the risk of progression in The Canadian Kidney Cancer Information System including 1448 clinically defined T1 RCC cases. Overall, 134 patients had disease upstaging to pT3a ( $n=66$ PN and $n=68 \mathrm{PN}$ ). After median follow-up of $23 \mathrm{mo}$, the 3-yr RFS was 76\% in upstaged patients compared to $93 \%$ in those with no upstaging $(p<0.001)$. Unfortunately, multivariable analyses comparing the effect of PN versus $\mathrm{RN}$ in the subgroup of patients with upstaging could not be performed because of the limited sample size [14]. Jeldres and colleagues [22] performed a multi-institutional matched comparison between PN $(n=30)$ and RN $(n=63)$ cases and found no significant cancer-specific survival differences after PN for pT3a lesions (HR 2.5; $p=0.9$ ). Owing to the small cohort size, a subgroup analysis of cT1N0M0 patients could not be undertaken.

Subgroup analyses for cT1 patients are rarely reported in the literature, and are exclusively in small cohorts of patients. Oh et al. [12] carried out a retrospective comparison of PN versus RN for patients with pT3a M0 RCC who had undergone surgery at five institutions in Korea (2000-2010). A subgroup analysis of 63 cT1a patients upgraded to pT3a revealed similar RFS rates between $\mathrm{PN}$ and $\mathrm{RN}$ groups.

To the best of our knowledge, the present study has evaluated the largest patient cohort available to date for the current topic. We are unaware of previous reports based on larger patient samples or of any previous analysis that relied on propensity-score matching of cohorts. Several questions might be raised by the current study. First, although future investigations are needed to draw a definitive conclusion, it might be asked whether the use of frozen sections during PN is needed in patients with clinically low-risk, organ-confined, small renal masses when similar oncological outcomes might be expected regardless of the surgical procedure. Second, it might be hypothesised that RN might not necessary in patients with unexpected nonorgan-confined disease at final pathology. Third, it appears that the surgical procedure should not be a factor when assessing the most adequate follow-up schedule after surgery. Unfortunately, all those hypotheses can only be formally validated in prospective settings, which are unachievable at present.

Our study is not devoid of limitations. First, the retrospective nature must be considered. As a consequence, it is conceivable that unmeasured variables for patient characteristics might have affected the results. Second, the RN group comprised individuals with larger and possibly more complex tumours, for which surgical challenges might have influenced the decision to perform RN instead of PN. In addition, PN patients were more represented in the later years of the study. However, year of surgery was included in our multivariable analyses to adjust for potential biases related to different lengths of follow-up. Third, the lack of central review for imaging and pathology specimen evaluation must also be considered. Specifically, the ability to correctly perform an adequate clinical staging depends on the imaging technique (computed tomography vs magnetic resonance imaging), the experience of the radiologist, and the protocols used at each institution.

\section{Conclusions}

Using a large multi-institutional cohort of cT1N0M0 RCC patients, we investigated whether PN might undermine cancer control when unanticipated pT3a disease is found at final pathology. In this specific scenario, despite the presence of unexpected non-organ-confined disease, PN does not seem to jeopardise cancer control.

Author contributions: Umberto Capitanio had full access to all the data in the study and takes responsibility for the integrity of the data and the accuracy of the data analysis.

Study concept and design: Capitanio.

Acquisition of data: Capitanio, Stewart, Rodriguez Faba, Klatte, Akdogan, Roscigno, Marszalek, Dell’Oglio, Zaffuto, Salagierski, Ouzaid, Lingard, Carini, Mir, Montorsi, Da Pozzo, Stief, Minervini, Brookman-May.

Analysis and interpretation of data: Capitanio, Dell'Oglio, Zaffuto.

Drafting of the manuscript: Capitanio, Stewart, Klatte, Dell'Oglio, Mir, Brookman-May.

Critical revision of the manuscript for important intellectual content: Capitanio, Stewart, Rodriguez Faba, Klatte, Akdogan, Roscigno, Marszalek, Dell'Oglio, Zaffuto, Salagierski, Ouzaid, Lingard, Carini, Mir, Montorsi, Da Pozzo, Stief, Minervini, Brookman-May.

Statistical analysis: Capitanio, Dell'Oglio, Zaffuto. Obtaining funding: None.

Administrative, technical, or material support: None.

Supervision: Capitanio, Stewart, Rodriguez Faba, Klatte, Akdogan, Roscigno, Marszalek, Dell'Oglio, Zaffuto, Salagierski, Ouzaid, Lingard, Carini, Mir, Montorsi, Da Pozzo, Stief, Minervini, Brookman-May. Other: None.

Financial disclosures: Umberto Capitanio certifies that all conflicts of interest, including specific financial interests and relationships and affiliations relevant to the subject matter or materials discussed in the manuscript (eg, employment/affiliation, grants or funding, consultancies, honoraria, stock ownership or options, expert testimony, royalties, or patents filed, received, or pending), are the following: None.

Funding/Support and role of the sponsor: None.

\section{References}

[1] Capitanio U, Montorsi F. Renal cancer. Lancet 2016;387:894-906. http://dx.doi.org/10.1016/S0140-6736(15)00046-X.

[2] Ljungberg B, Bensalah K, Canfield S, et al. EAU guidelines on renal cell carcinoma: 2014 update. Eur Urol 2015;67:913-24. http://dx. doi.org/10.1016/j.eururo.2015.01.005.

[3] National Comprehensive Cancer Network NCCN guidelines: kidney cancer version 3. For Washington, PA: NCCN; 2015.

[4] Campbell SC, Novick AC, Belldegrun A, et al. Guideline for management of the clinical T1 renal mass. J Urol 2009;182:1271-9. http:// dx.doi.org/10.1016/j.juro.2009.07.004. 
[5] Capitanio U, Terrone C, Antonelli A, et al. Nephron-sparing techniques independently decrease the risk of cardiovascular events relative to radical nephrectomy in patients with a T1a-T1b renal mass and normal preoperative renal function. Eur Urol 2015; 67:683-9. http://dx.doi.org/10.1016/j.eururo.2014.09.027.

[6] Scosyrev E, Messing EM, Sylvester R, Campbell S, Van Poppel H. Renal function after nephron-sparing surgery versus radical nephrectomy: results from EORTC randomized trial 30904. Eur Urol 2014;65:372-7. http://dx.doi.org/10.1016/j.eururo.2013.06.044.

[7] Lane BR, Demirjian S, Derweesh IH, et al. Survival and functional stability in chronic kidney disease due to surgical removal of nephrons: importance of the new baseline glomerular filtration rate. Eur Urol 2015;68:996-1003. http://dx.doi.org/10.1016/j. eururo.2015.04.043.

[8] Shin TY, Komninos C, Kim DW, et al. A novel mathematical model to predict the severity of postoperative functional reduction before partial nephrectomy: the importance of calculating resected and ischemic volume. J Urol 2015;193:423-9. http://dx.doi.org/10.1016/ j.juro.2014.07.084.

[9] Zhang Z, Zhao J, Dong W, et al. Acute ipsilateral renal dysfunction after partial nephrectomy in patients with a contralateral kidney: spectrum score to unmask ischemic injury. Eur Urol 2016;70:692-8. http://dx.doi.org/10.1016/j.eururo.2016.04.015.

[10] Capitanio U, Larcher A, Terrone C, et al. End-stage renal disease after renal surgery in patients with normal preoperative kidney function: balancing surgical strategy and individual disorders at baseline. Eur Urol 2016;70:558-61. http://dx.doi.org/10.1016/j.eururo.2016.03. 023.

[11] Van Poppel H, Da Pozzo L, Albrecht W, et al. A prospective, randomised EORTC intergroup phase 3 study comparing the oncologic outcome of elective nephron-sparing surgery and radical nephrectomy for low-stage renal cell carcinoma. Eur Urol 2011;59:543-52. http://dx.doi.org/10.1016/j.eururo.2010.12.013.

[12] Oh JJ, Byun S-S, Lee SE, et al. Partial nephrectomy versus radical nephrectomy for non-metastatic pathological T3a renal cell carcinoma: a multi-institutional comparative analysis. Int J Urol 2014; 21:352-7. http://dx.doi.org/10.1111/iju.12283.

[13] Gorin MA, Ball MW, Pierorazio PM, et al. Outcomes and predictors of clinical T1 to pathological T3a tumor up-staging after robotic partial nephrectomy: a multi-institutional analysis. J Urol 2013;190:190711. http://dx.doi.org/10.1016/j.juro.2013.06.014.

[14] Nayak JG, Patel P, Saarela O, et al. Pathological upstaging of clinical T1 to pT3a renal cell carcinoma: a multi-institutional analysis of short-term outcomes. Urology 2016;94:154-60. http://dx.doi.org/ 10.1016/j.urology.2016.03.029.

[15] Kutikov A, Smaldone MC, Uzzo RG. Partial versus radical nephrectomy: balancing nephrons and perioperative risk. Eur Urol 2013;64:607-9. http://dx.doi.org/10.1016/j.eururo.2013.01.020.

[16] Chevinsky M, Imnadze M, Sankin A, et al. Pathological stage T3a significantly increases disease recurrence across all tumor sizes in renal cell carcinoma. J Urol 2015;194:310-5. http://dx.doi.org/10. 1016/j.juro.2015.02.013.

[17] Ramaswamy K, Kheterpal E, Pham H, et al. Significance of pathologic T3a upstaging in clinical T1 renal masses undergoing nephrectomy. Clin Genitourin Cancer 2015;13:344-9. http://dx.doi.org/10.1016/j. clgc.2015.01.001.

[18] Ball MW, Gorin MA, Bhayani SB, et al. Preoperative predictors of malignancy and unfavorable pathology for clinical T1a tumors treated with partial nephrectomy: a multi-institutional analysis. Urol Oncol 2015;33:112.e9-112.e14. http://dx.doi.org/10.1016/j. urolonc.2014.11.003.

[19] Sokhi HK, Mok WY, Patel U. Stage T3a renal cell carcinoma: staging accuracy of $\mathrm{CT}$ for sinus fat, perinephric fat or renal vein invasion. $\mathrm{Br}$ J Radiol 2015;88:20140504. http://dx.doi.org/10.1259/bjr. 20140504.

[20] Schiavina R, Borghesi M, Chessa F, et al. The prognostic impact of tumor size on cancer-specific and overall survival among patients with pathologic T3a renal cell carcinoma. Clin Genitourin Cancer 2015;13:e235-41. http://dx.doi.org/10.1016/j.clgc.2014.06.011.

[21] Brookman-May S, Johannsen M, May M, et al. Difference between clinical and pathologic renal tumor size, correlation with survival, and implications for patient counseling regarding nephron-sparing surgery. Am J Roentgenol 2011;197:1137-45. http://dx.doi.org/10. 2214/AJR.11.6534.

[22] Jeldres C, Patard J-J, Capitanio U, et al. Partial versus radical nephrectomy in patients with adverse clinical or pathologic characteristics. Urology 2009;73:1300-5. http://dx.doi.org/10.1016/j. urology.2008.08.492. 